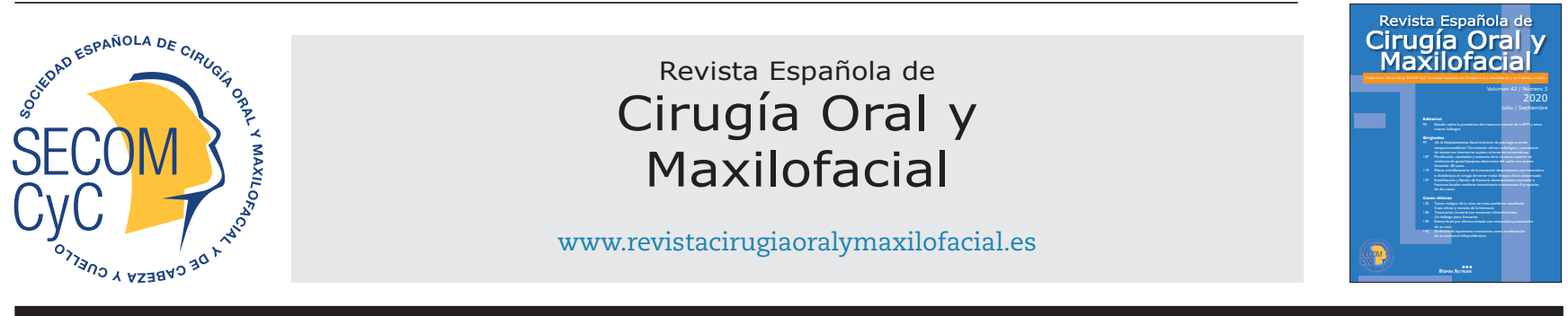

\title{
Original
}

\section{Efecto antinflamatorio de la asociación dexametasona con ketorolaco o diclofenaco en cirugía de tercer molar. Ensayo clínico aleatorizado}

\section{Victor Chumpitaz-Cerrate ${ }^{1,2^{*}}$, Carlos Erickson Capillo-Carrillo ${ }^{2}$ y Lesly Chávez-Rimache ${ }^{3}$}

${ }^{1}$ Facultad de Ciencias de la Salud, Laboratorio de Farmacología, Universidad Científica del Sur, Lima, Perú. ${ }^{2}$ Facultad de Odontología, Universidad Nacional Mayor de San Marcos, Lima, Perú. ${ }^{3}$ Facultad de Odontología, Laboratorio de Farmacología, Universidad Nacional Mayor de San Marcos, Lima, Perú

\section{INFORMACIÓN DEL ARTÍCULO}

Historia del artículo:

Recibido: 5 de febrero de 2019

Aceptado: 15 de febrero de 2019

Palabras clave:

Dexametasona, diclofenaco, ketorolaco, analgesia preventiva, cirugía de tercer molar.

\section{R E S U M E N}

Objetivo: Evaluar el efecto antinflamatorio de la asociación dexametasona con diclofenaco y la asociación dexametasona con ketorolaco en cirugía de tercer molar mandibular.

Material y método: Ensayo clínico aleatorizado doble ciego que incluyó 75 pacientes que se dividieron aleatoriamente en tres grupos: el grupo DK recibió la asociación dexametasona con ketorolaco; el grupo DD, la asociación dexametasona con diclofenaco y el grupo control $\mathrm{D}$, solo dexametasona; todos por vía intramuscular, treinta minutos antes de la cirugía. El efecto antinflamatorio se determinó por la evaluación del dolor y la tumefacción postquirúrgicos. El dolor se evaluó mediante la escala visual análoga y el consumo total de analgésicos. La tumefacción se evalúo mediante las medidas de tumefacción facial y la apertura bucal. Resultados: El grupo DD evidenció menor intensidad de dolor a las 4, 6, 12, 24 y 48 horas en comparación a los otros grupos de tratamiento $(p<0,05)$. El consumo total de analgésicos fue significativamente menor en el grupo DD $(1,20 \pm 0,3)$ en comparación con los otros grupos de tratamiento $(p=0,002)$. La tumefacción facial fue similar en todos los grupos de estudio $(p>0,05)$. El grupo DD evidenció una significativa mayor apertura bucal solamente a las 48 horas postoperatorias en comparación a los otros grupos de estudio $(p=0,01)$.

Conclusiones: La administración preoperatoria de la asociación dexametasona con diclofenaco presenta una significativa mayor actividad analgésica en comparación a la administración preoperatoria de la asociación dexametasona con ketorolaco. Sin embargo, no hay diferencia significativa con relación a la tumefacción facial.

\footnotetext{
*Autor para correspondencia:

Correo electrónico: vchumpitaz@ucientifica.edu.pe (Victor Chumpitaz Cerrate).
} 
Anti-inflammatory effect of the dexamethasone association with ketorolac or diclofenac in third molar surgery. Randomized clinical trial

\section{A B S T R A C T}

Keywords:

Dexamethasone, diclofenac, ketorolac, preventive analgesia, third molar surgery.
Objective: To evaluate the antiinflammatory effect of the association dexamethasone with diclofenac and the association dexamethasone with ketorolac in mandibular third molar surgery.

Material and method: Randomized double blind clinical trial that included 75 patients who were randomly divided into three groups: the DK group received the dexamethasone association with ketorolac; the DD group, the dexamethasone association with diclofenac and the control group D, only dexamethasone; all intramuscularly, thirty minutes before surgery. The anti-inflammatory effect was determined by the evaluation of postoperative pain and swelling. The pain was evaluated by means of the analogous visual scale and the total analgesic consumption. Swelling was assessed by measures of facial swelling and mouth opening. Results: The DD group showed less pain intensity at $4 \mathrm{~h}, 6 \mathrm{~h}, 12 \mathrm{~h}, 24 \mathrm{~h}$ and $48 \mathrm{~h}$ compared to the other treatment groups $(p<0.05)$. Total analgesic consumption was significantly lower in the DD group $(1.20 \pm 0.3)$ compared to the other treatment groups; $p=0.002$. Facial swelling was similar in all study groups $(\mathrm{p}>0.05)$. The DD group showed a significant greater oral opening only at 48 hours postoperatively compared to the other study groups $(p=0.01)$.

Conclusions: The preoperative administration of the dexamethasone association with diclofenac has a significantly greater analgesic activity compared to the preoperative administration of the dexamethasone association with ketorolac. However, there is no significant difference in relation to facial swelling.

\section{INTRODUCCIÓN}

La remoción quirúrgica del tercer molar es un modelo ampliamente estudiado para evaluar la eficacia de analgésicos y antinflamatorios ${ }^{1,2}$. El éxito del procedimiento se basa en el control del dolor e inflamación postquirúrgicos, los cuales son una preocupación constante de todo cirujano oral y maxilofacial ${ }^{2}$.

La analgesia preventiva en la exodoncia de terceros molares ha mostrado resultados contradictorios ${ }^{3}$. La monoterapia con antinflamatorios no esteroideos (AINE), corticoides $u$ opioides, puede resultar insuficiente para conseguir óptimas condiciones postoperatorias. Por ello, el uso de asociaciones es una alternativa para disminuir el dolor, tumefacción facial y trismo postquirúrgicos 4,5 .

El diclofenaco es un AINE que por sus características farmacocinéticas y farmacodinámicas controla adecuadamente la tumefacción facial y el dolor postquirúrgicos ${ }^{3}$. El ketorolaco es otro AINE que ha evidenciado un adecuado efecto analgésico durante las primeras horas del postquirúrgico inmediato ${ }^{6}$.

La asociación dexametasona con diclofenaco ha evidenciado efectos sinérgicos, analgésicos y antinflamatorios. Otra asociación frecuentemente empleada es dexametasona con ketorolaco (dexametasona es un potente antinflamatorio y ketorolaco es un potente analgésico); sin embargo, no se han realizado ensayos clínicos para evaluar su eficacia combinada en la cirugía de tercer molar.

La dexametasona no posee efecto analgésico intrínseco, pero al inhibir la producción de mediadores proinflamatorios como interleuquinas (IL-1 $\beta$ e IL-6), factor de necrosis tumo- ral (TNF) y óxido nítrico (NO), produce un efecto analgésico indirecto al controlar la extensión del proceso inflamatorio, disminuyendo la intensidad de las secuelas postoperatorias como el dolor ${ }^{7}$.

El objetivo del presente estudio fue evaluar el efecto antinflamatorio de la administración preoperatoria de la asociación dexametasona con diclofenaco en comparación a la administración preoperatoria de la asociación dexametasona con ketorolaco en cirugía de tercer molar mandibular.

\section{MATERIAL Y MÉTODO}

Ensayo clínico aleatorizado acorde con la declaración CONSORT $^{8}$. La muestra estuvo conformada por 75 pacientes adultos jóvenes, de 18 a 30 años, candidatos a recibir exodoncia de tercer molar mandibular y que cumplieron los criterios de inclusión del Servicio de Cirugía Oral del Instituto de Salud Oral de la Fuerza Aérea del Perú (FAP) durante el año 2016.

La muestra fue aleatorizada por un muestreo probabilístico aleatorio simple. Para el cálculo del tamaño muestral se consideró un nivel de confianza del 95 \% y un poder estadístico del $80 \%$.

\section{Consideraciones bioéticas}

El protocolo del estudio fue desarrollado de acuerdo con la declaración de Helsinki y fue aprobado por el comité de ética del Hospital Central de la Fuerza Aérea del Perú (FAP)ํ. 


\section{Criterios de selección}

Los criterios de inclusión fueron pacientes ASA I, de 18 a 30 años, con un tercer molar mandibular clase II o III y posición B o C de Pell y Gregory, que no sean alérgicos a los anestésicos locales, AINE y/o corticoides. Los criterios de exclusión fueron pacientes fumadores, gestantes o con infección odontogénica.

El investigador principal entregó el consentimiento informado a cada paciente, luego se realizó una segunda fase de selección para determinar el índice de dificultad quirúrgica según Gbotolorum ${ }^{10}$, que evalúa factores como edad, índice de masa corporal, profundidad del tipo de elevación y curvatura de las raíces. Para el estudio se seleccionaron a los pacientes que presenten dificultad moderada (puntuación entre 7 y 9).

Posterior a ello, se distribuyeron aleatoriamente en tres grupos de 25 integrantes, que recibieron los siguientes tratamientos:

- Grupo DK: dexametasona $4 \mathrm{mg} / 2 \mathrm{ml}$ y ketorolaco $60 \mathrm{mg} / 2 \mathrm{ml}$, ambos administrados vía intramuscular, treinta minutos antes de la cirugía.

- Grupo DD: dexametasona $4 \mathrm{mg} / 2 \mathrm{ml}$ y diclofenaco $75 \mathrm{mg} / 3 \mathrm{ml}$, ambos administrados vía intramuscular, treinta minutos antes de la cirugía.

- Grupo D: dexametasona $4 \mathrm{mg} / 2 \mathrm{ml}$ vía intramuscular, treinta minutos antes de la cirugía.

No existió un grupo control con placebo debido a que el protocolo quirúrgico del Instituto de Salud Oral de la FAP indica el uso de medicación preventiva para todas las exodoncias quirúrgicas de terceros molares mandibulares.

Un especialista en cirugía oral y maxilofacial realizó todos los procedimientos quirúrgicos e ignoraba la medicación asignada a cada paciente. Se empleó una técnica quirúrgica estandarizada.

Se realizó la asepsia y antisepsia del campo operatorio con iodopovidona. Luego se realizó el bloqueo del nervio dentario inferior con lidocaína 2 \% con adrenalina 1:80000 (Xylestesin) ${ }^{\circledR}$. Se realizó una primera incisión desde el borde anterior de la rama ascendente hasta la zona retromolar continuando con una incisión sulcular; se decoló el colgajo y se realizó la osteotomía y odontosección con fresa para hueso n. ${ }^{\circ} 16$ (Maillefer) ${ }^{\circledR}$. Se realizó un lavado con suero fisiológico y se reposicionó el colgajo. Se consideró la duración de la cirugía como el tiempo transcurrido desde que se realizó la primera incisión hasta que se colocó el último punto de sutura.

Posterior al procedimiento quirúrgico se le brindaron al paciente instrucciones escritas. Como farmacoterapia se le indicó: amoxicilina 500 mg, 1 cápsula cada 8 horas por 5 días y la asociación tramadol 37,5 mg con paracetamol $325 \mathrm{mg}$, 1 tableta v.o. cada 8 horas por 3 días y luego condicionado al dolor (hasta un máximo de tres tabletas al día, separadas cada 8 horas, por un máximo de 2 días). Se le entregó al paciente una ficha de recolección de datos para que registrara los valores de intensidad del dolor y consumo total de analgésicos.

El efecto antinflamatorio se determinó por la evaluación del dolor y la tumefacción.

El dolor se evalúo mediante la escala visual análoga (EVA) y el consumo total de analgésicos.

\section{Intensidad del dolor según la escala visual análoga (EVA)}

La escala visual análoga fue representada gráficamente por una línea recta de $100 \mathrm{~mm}$, con valores extremos de $0 \mathrm{y}$ 100 (0 "ausencia de dolor" y 100 "el máximo dolor imaginable"). Se solicitó a cada paciente que marcara un punto ubicado entre 0 y 100 para indicar la intensidad del dolor que percibía después de $4,6,12,24$ y 48 horas posteriores a la finalización de la cirugía.

\section{Consumo total de analgésicos}

Se solicitó a cada paciente que registrara la cantidad de tabletas (tramadol 37,5 mg con paracetamol $325 \mathrm{mg}$ ) que consumió durante los 7 días postoperatorios.

La tumefacción se evalúo mediante las medidas de tumefacción facial y la apertura bucal.

\section{Medidas de tumefacción facial}

Se ubicaron los puntos anatómicos: tragus, ángulo externo del ojo, ala de la nariz, comisura labial, pogonion blando y gonion. Se registró la suma de las distancias faciales: tragus-ángulo externo del ojo, tragus-comisura labial, tragus-pogonion blando y tragus-gonion, mediante un vernier electrónico en el preoperatorio, postoperatorio inmediato, segundo y séptimo día postoperatorios.

\section{Apertura bucal}

Se evaluó, para cada paciente, la distancia entre los bordes incisales de los incisivos centrales superior e inferior. Se registraron las medidas en el preoperatorio, postoperatorio inmediato, segundo y séptimo día postoperatorios.

\section{Análisis de datos}

El análisis e interpretación de datos se realizó con el programa estadístico SPSS versión 22.0. Se empleó la prueba Shapiro Wilk para determinar la normalidad de las variables. Para las variables paramétricas se utilizó la prueba ANOVA con la prueba post hoc Tukey para la comparación múltiples de medias. Se consideró un nivel de confianza del $95 \%$ y se realizó el análisis por intención de tratar.

\section{RESULTADOS}

Del total de participantes ( $\mathrm{n}=75), 2$ no recibieron la cirugía porque iban a empezar su tratamiento de ortodoncia y 2 abandonaron el estudio porque no asistieron a sus citas de control. El análisis fue por intención de tratar (Figura 1).

La edad media de los participantes fue 20,87 $\pm 2,20$ años. El $46,7 \%$ fueron mujeres y el 53,3\%, varones. 


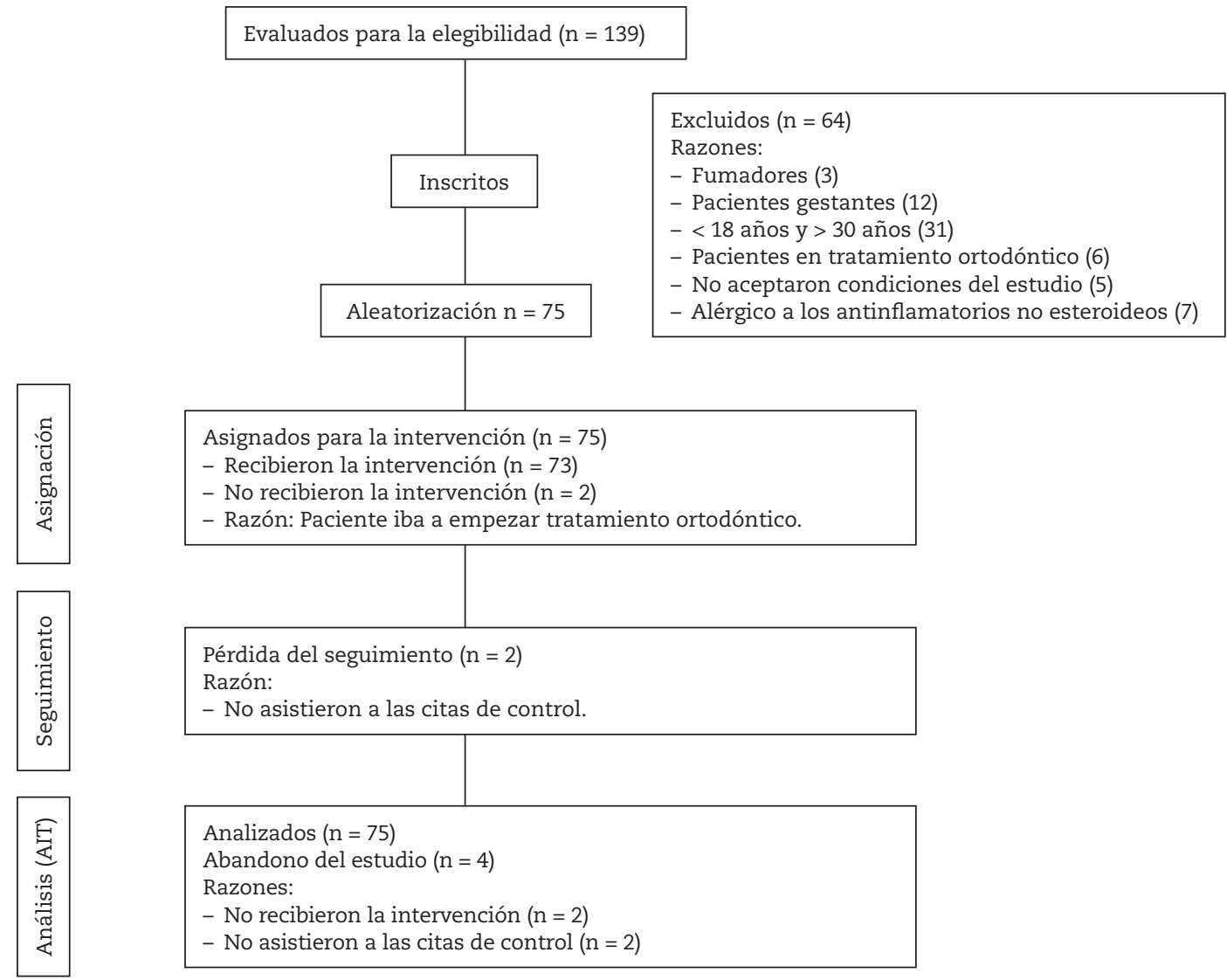

Figura 1. Diagrama de flujo de reclutamiento de pacientes en este estudio acorde con la declaración CONSORT. AIT: Análisis por intención de tratar.

El tiempo quirúrgico en el grupo DK fue 24,92 $\pm 2,58$ minutos; en el grupo DD fue $24,20 \pm 3,67$ minutos y en el grupo D fue $24,56 \pm 1,87$ minutos; $p=1,13$. El índice de dificultad quirúrgica para el grupo DK fue 8,14 $\pm 1,32$; el del grupo DD fue $7,91 \pm 1,74$ y para el grupo D fue 8,36 $\pm 0,89 ; p=0,097$.

\section{Intensidad del dolor según EVA (Figura 2)}

La intensidad del dolor a las 4 horas en el grupo DK fue $16,78 \pm 2,15$ (IC $95 \%=16,28-17,27$ ), en el grupo DD fue $12,44 \pm$ 1,51 (IC $95 \%=11,78-13,02$ ) y en el grupo D fue $27,89 \pm 4,91$ (IC $95 \%=26,76-29,07) ; p=0,001$.

La intensidad del dolor a las 6 horas en el grupo DK fue $25,29 \pm 5,12$ (IC $95 \%=21,11-27,46$ ), en el grupo DD fue $24,04 \pm$ 4,78 (IC $95 \%=20,94-27,13$ ) y en el grupo D fue $40,35 \pm 8,38$ (IC $95 \%=36,41-44,27) ; p=0,002$.

La intensidad del dolor a las 12 horas en el grupo DK fue $27,3 \pm 6,13$ (IC $95 \%=23,98-29,75$ ), en el grupo DD fue 19,53 \pm 4,84 (IC $95 \%=17,41-22,64$ ) y en el grupo D fue $50,91 \pm 8,71$ (IC $95 \%=46,9-54,91) ; \mathrm{p}=0,001$.

La intensidad del dolor a las 24 horas en el grupo DK fue $31,41 \pm 5,72$ (IC $95 \%=28,09-34,72$ ), en el grupo DD fue 15,66 \pm 3,48 (IC $95 \%=12,85-18,46$ ) y en el grupo D fue $42,79 \pm 6,13$ (IC $95 \%=39,37-46,20) ; p=0,002$.

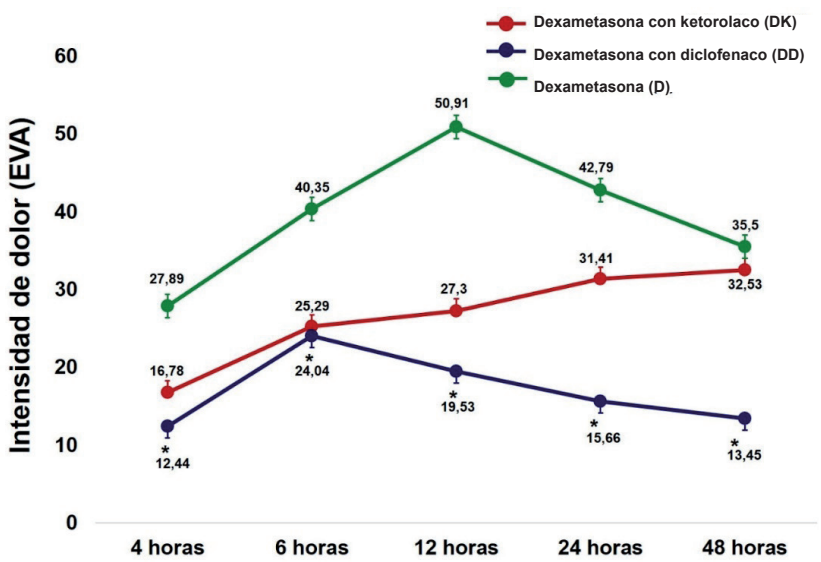

Figura 2. Intensidad de dolor (EVA) según grupo de estudio. Cada punto representa la media \pm desviación estándar. *Prueba estadística ANOVA; $\mathrm{p}<0,05$.

La intensidad del dolor a las 48 horas en el grupo DK fue $32,53 \pm 2,97$ (IC $95 \%=29,84-35,21$ ), en el grupo DD fue 13,45 \pm 2,12 (IC $95 \%=11,96-15,93$ ) y en el grupo D fue 35,50 $\pm 3,75$ (IC $95 \%=32,63-38,36) ; p=0,003$. 


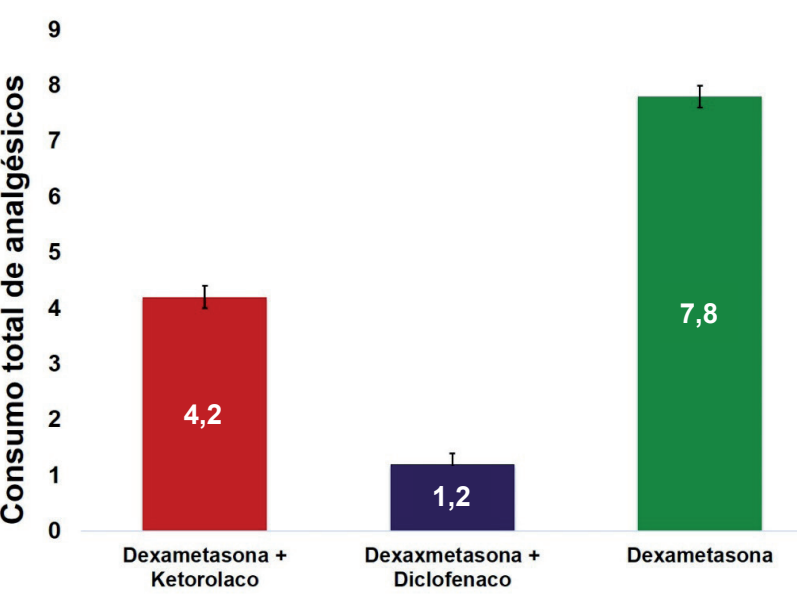

Figura 3. Consumo total de analgésicos según grupo de estudio.

Prueba estadística ANOVA; $\mathrm{p}<0,05$.

Con la prueba post hoc Tukey solamente se observó diferencia significativa entre los grupos DK y DD, a las 24 y 48 horas ( $p=0,001$ y $p=0,004$, respectivamente).

\section{Consumo total de analgésicos (Figura 3)}

El consumo total de tabletas analgésicas en el grupo DK fue 4,20 $\pm 1,3$ (IC $95 \%=3,64-4,76$ ), en el grupo DD fue $1,20 \pm 0,3$ (IC $95 \%=0,82-1,58$ ) y en el grupo D fue 7,8 $\pm 0,91$ (IC $95 \%=7,42-8,18) ; \mathrm{p}=0,002$.

Con la prueba post hoc Tukey se evidenció diferencia significativa entre todos los pares de grupos evaluados.

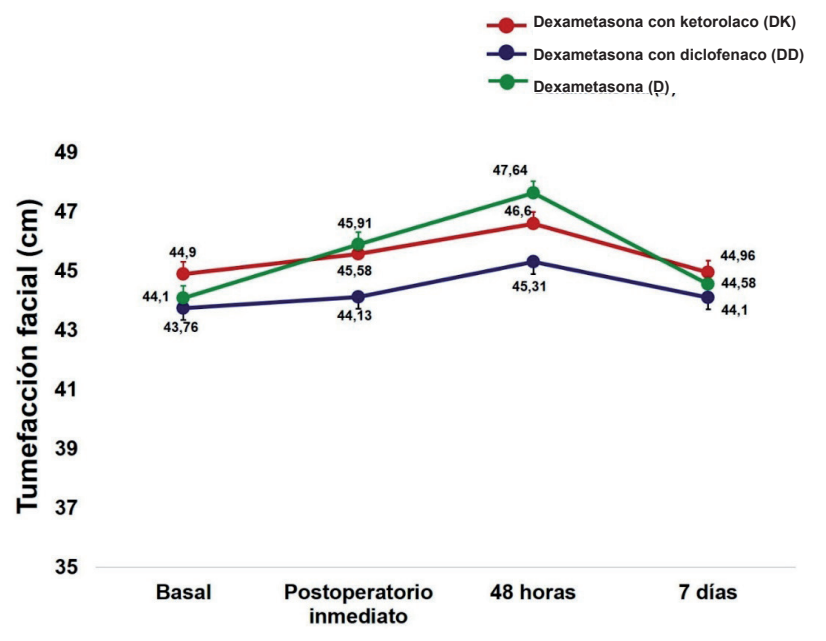

Figura 4. Medias de tumefacción facial según grupo de estudio. Cada punto representa la media \pm desviación estándar.

Prueba estadística ANOVA; $\mathrm{p}>0,05$.

\section{Medidas de tumefacción facial (Figura 4)}

El valor preoperatorio en el grupo DK fue $44,9 \pm 1,8 \mathrm{~cm}$ (IC $95 \%=43,89-45,76$ ); en el grupo DD fue 43,76 $\pm 1,51 \mathrm{~cm}$ (IC $95 \%$ $=43,12-45,31)$ y en el grupo D fue $44,15 \pm 1,17 \mathrm{~cm}$ (IC $95 \%=43,71-$ 44,86); $\mathrm{p}=0,675$.

El valor postoperatorio inmediato en el grupo DK fue $45,58 \pm 1,9 \mathrm{~cm}$ (IC $95 \%=44,36-46,21$ ), en el grupo DD fue 44,13 $\pm 1,54 \mathrm{~cm}$ (IC $95 \%=43,04-45,31)$ y en el grupo D fue 45,91 \pm $1,71 \mathrm{~cm}$ (IC $95 \%=44,76-46,4) ; \mathrm{p}=0,677$.

El valor al segundo día postoperatorio en el grupo DK fue $46,67 \pm 1,79 \mathrm{~cm}$ (IC $95 \%=45,78-47,91$ ), en el grupo DD fue 45,34 $\pm 1,58 \mathrm{~cm}$ (IC $95 \%=44,21-46,8)$ y en el grupo D fue $47,64 \pm 0,97$ $\mathrm{cm}$ (IC $95 \%=46,9-48,2) ; \mathrm{p}=0,36$.

El valor al séptimo día postoperatorio en el grupo DK fue 44,96 $\pm 1,49 \mathrm{~cm}$ (IC $95 \%=44,16-45,61)$, en el grupo DD fue $44,15 \pm 2,13 \mathrm{~cm}$ (IC $95 \%=43,8-45,6$ ) y en el grupo D fue $44,58 \pm 0,87 \mathrm{~cm}($ IC $95 \%=43,96-45,01) ; p=0,41$.

\section{Apertura bucal (Figura 5)}

La apertura bucal preoperatoria en el grupo DK fue 49,33 \pm $4,87 \mathrm{~mm}$ (IC $95 \%=48,20-50,95$ ), en el grupo DD fue 49,23 $\pm 4,07 \mathrm{~mm}$ (IC $95 \%=47,97-50,56)$ y en el grupo D fue 49,59 \pm 1,36 mm (IC $95 \%=48,72-50,02$ ); $\mathrm{p}=0,96$.

La apertura bucal en el postoperatorio inmediato en el grupo DK fue 40,53 $\pm 4,96 \mathrm{~mm}$ (IC $95 \%=39,38-41,67$ ), en el grupo DD fue $41,03 \pm 3,13 \mathrm{~mm}$ (IC $95 \%=40,17-42,05)$ y en el grupo D fue 41,04 $\pm 1,07 \mathrm{~mm}$ (IC $95 \%=40,79-41,30$ ); $\mathrm{p}=0,89$.

La apertura bucal al segundo día postoperatorio en el grupo DK fue $27,22 \pm 4,07 \mathrm{~mm}$ (IC $95 \%=26,28-28,15$ ), en el grupo DD fue $35,07 \pm 4,95 \mathrm{~mm}$ (IC $95 \%=33,93-36,20$ ) y en el grupo D fue $27,02 \pm 4,77 \mathrm{~mm}$ (IC $95 \%=25,92-28,11$ ); $\mathrm{p}=0,01$.

La apertura bucal al séptimo día postoperatorio en el grupo DK fue 40,62 $\pm 6,87 \mathrm{~mm}$ (IC $95 \%=39,03-42,20$ ), en el grupo

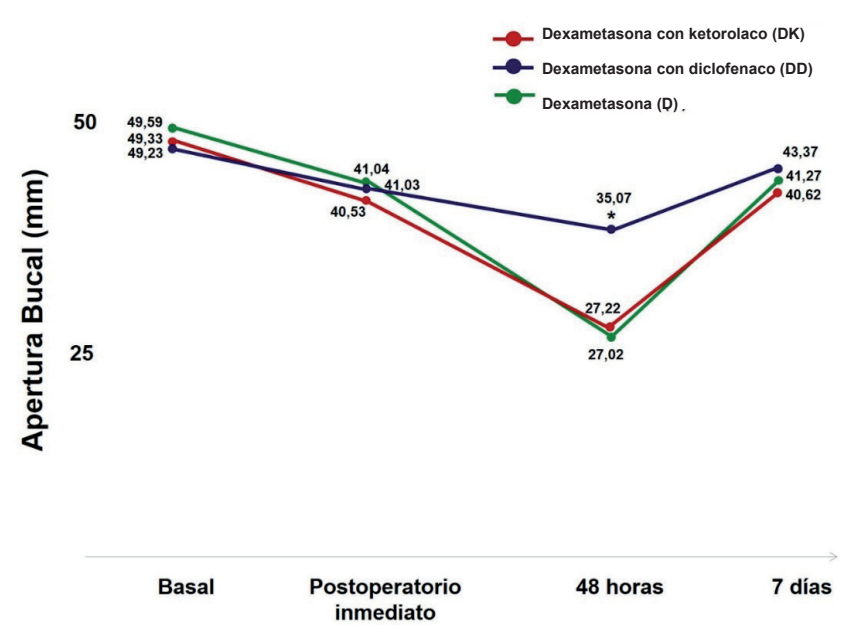

Figura 5. Medias de apertura bucal según grupo de estudio. Cada punto representa la media \pm desviación estándar.

Prueba estadística ANOVA; $\mathrm{p}<0,05$. 
DD fue 43,37 $\pm 4,72 \mathrm{~mm}$ (IC $95 \%=42,28-44,45$ ) y en el grupo D fue 41,27 $\pm 4,87 \mathrm{~mm}$ (IC $95 \%=40,14-42,39$ ); $\mathrm{p}=0,48$.

Con la prueba post hoc Tukey solamente se observó diferencia significativa al segundo día postoperatorio entre los grupos DK y DD, así como entre los grupos DD y D ( $p=0,009$ y $p=0,005$, respectivamente).

\section{DISCUSIÓN}

La exodoncia de terceros molares es un procedimiento invasivo capaz de desencadenar niveles variables de dolor producidos por la inflamación ${ }^{11}$. Esto contribuye al empleo de este modelo clínico para evaluar la eficacia de los analgésicos y antinflamatorios prescritos convencionalmente ${ }^{12,13}$.

En el presente trabajo de investigación se encontró que la administración preoperatoria de la asociación dexametasona con diclofenaco demostró tener un efecto analgésico significativamente superior en comparación a la administración preoperatoria de la asociación dexametasona con ketorolaco.

El ketorolaco presenta un efecto analgésico potente y algunos autores como Grant y cols. ${ }^{14}$ e Isiordia-Espinoza y cols. ${ }^{6}$ mencionan que modula los receptores opioides y estimula la liberación de óxido nítrico. El ketorolaco es un inhibidor preferencial de la cicloxigenasa 1 (COX-1), que se expresa constitutivamente y participa en la homeostasis de los procesos fisiológicos; sin embargo, también está presente durante el inicio del dolor posterior a cirugía de tercer molar ${ }^{15}$.

El diclofenaco es un inhibidor preferencial de la ciclooxigenasa 2 (COX-2), la cual aumenta su expresión en procesos inflamatorios, incrementando la liberación de prostaglandina E2 (PGE2), TNF- $\alpha$ e IL-1 $\beta$, mediadores que sensibilizan a las neuronas aferentes provocando hiperalgesia. Además, inhibe a la lipooxigenasa, activa el sistema descendente inhibitorio del dolor y se acumula en el tejido inflamado ${ }^{16}$. Se ha referido que la asociación dexametasona con diclofenaco presenta efecto sinérgico en cirugía de tercer molar, por lo que se esperaría un resultado exitoso en todas las variables evaluadas. Sin embargo, la tumefacción facial presentó un comportamiento clínico similar en todos los grupos de la presente investigación, incluso en el grupo que recibió solo dexametasona. Bamgbose y cols. ${ }^{17}$ encontraron que la asociación diclofenaco con dexametasona produjo mayor reducción del dolor e inflamación, los días 1 y 2 postoperatorios, en comparación con diclofenaco ( $p<0,05$ y p > 0,05, respectivamente), sin efectos adversos al usar la asociación. Sin embargo, Hernández-Díaz y cols. ${ }^{18}$ realizaron un estudio de casos y controles para estimar el riesgo de complicaciones gastrointestinales asociadas al uso de corticoides y AINE, encontrando que el riesgo de hemorragia digestiva alta por el uso de solo AINE fue $\mathrm{OR}=4,0$; para el uso de solo corticoides fue $\mathrm{OR}=1,8$; y para el uso de corticoides asociados con AINE fue $\mathrm{OR}=8,9$. Recomiendan administrar antinflamatorios en monoterapia y en la dosis mínima efectiva para reducir el riesgo de complicaciones gastrointestinales. Asimismo, Chegini y cols. ${ }^{4}$, en su revisión sistemática, mencionan que el uso de la asociación corticoide con AINE puede aumentar el riesgo de gastrolesividad, por lo que recomiendan que se prescriba en una sola administración y en adultos jóvenes sanos.
Buyukkurt y cols. ${ }^{19}$ encontraron un significativo mayor efecto analgésico de la asociación prednisolona con diclofenaco en comparación a solo prednisolona, sin diferencia significativa con relación con la tumefacción facial.

En el presente estudio, la apertura bucal fue similar en todos los grupos de estudio, excepto a las 48 horas, cuando la asociación dexametasona con diclofenaco produjo una significativa mayor apertura bucal. Esto es muy relevante debido a que a las 48 horas se presenta el máximo nivel de tumefacción facial en los pacientes, afectando su calidad de vida ${ }^{2}$.

Por otro lado, a pesar de que la administración preoperatoria de ketorolaco puede producir sangrado intra y postoperatorio, en el presente estudio ningún paciente manifestó complicaciones hemorrágicas, lo cual coindice con lo referido por Isiordia-Espinoza y cols. ${ }^{14}$, Singh y cols. ${ }^{20}$ y Mony y cols. ${ }^{21}$.

Medeiros-Albuquerque y cols. ${ }^{22}$, en su revisión sistemática, encontraron que las metaloproteinasas de matriz (MMP) desempeñan un papel importante en la lesión inflamatoria aguda y su actividad está regulada por la liberación de PGE2, la cual es producida por la COX-2. Los AINE, al inhibir a la COX-2, disminuyen la expresión de MMP, incrementando su efecto antinflamatorio.

Kahn y cols. ${ }^{23}$ realizaron un análisis cuantitativo de COX-1 y COX-2 en un modelo de exodoncia de tercer molar, encontrando que la expresión del gen de la COX-2 evidenció una banda muy débil al inicio, pero fue incrementándose a medida que pasaba el tiempo y el gen de la COX-1 fue disminuyendo progresivamente a medida que pasaba el tiempo. Lee y cols. ${ }^{24}$ observaron a las 2 y 4 horas postoperatorias un aumento de la expresión génica de COX-2 y una disminución de COX-1, volviendo a los valores prequirúrgicos a las 48 horas. Estos hallazgos fueron similares a lo encontrado en el presente estudio: la asociación dexametasona con diclofenaco (inhibidor preferencial de COX-2) tuvo un efecto analgésico que fue incrementándose con el paso del tiempo; mientras la asociación dexametasona con ketorolaco (inhibidor preferencial de COX-1) tuvo un efecto analgésico menor que fue disminuyendo con el paso del tiempo.

La dexametasona presenta un efecto analgésico derivado de su potente efecto antinflamatorio. Simone y cols. ${ }^{2}$ encontraron que la dexametasona presentó un efecto analgésico superior al diclofenaco, sin diferencia significativa $(p=0,605)$. Esto puede atribuirse a que la dexametasona inhibe a la fosfolipasa A2 e inhibe al gen de la COX-2 ${ }^{24}$. Por este motivo, hay necesidad de investigar su efecto analgésico al asociarlo con fármacos como diclofenaco o ketorolaco.

Actualmente existen algunas limitaciones en el modelo experimental de cirugía de tercer molar debido al uso de analgésicos y antinflamatorios con diferentes dosis, vías de administración y distintos diseños metodológicos. Esto produce mucha heterogeneidad en los estudios, lo cual dificulta establecer protocolos analgésicos-antinflamatorios en cirugía de tercer molar mandibular. Una de las limitaciones del presente estudio fue que el diseño del estudio no fue a boca partida debido a la escasa cantidad de pacientes que acuden al Instituto de Salud Oral con indicación de exodoncia quirúrgica de ambos terceros molares mandibulares (derecho e izquierdo). Además, para determinar la tumefacción facial no 
se consideró el biotipo facial para la estandarización de los grupos de estudio según su patrón mesofacial, braquifacial o dolicofacial, esto podría haber sido un factor que haya influido en los resultados.

En base a la literatura revisada, se recomienda que en futuras investigaciones se considere realizar estudios a boca partida para minimizar la variabilidad entre los pacientes y evaluar la tumefacción facial, previa estandarización del biotipo facial, así como su medición con instrumentos más precisos como tomografías, ultrasonografías y pletismógrafos faciales. Según el grado de recomendación brindada por Oxford Centre for Evidence-Based Medicine (OCEBM) la presente investigación tiene un grado de recomendación $\mathrm{A}^{25}$.

\section{CONCLUSIONES}

La administración preoperatoria de la asociación dexametasona con diclofenaco presenta una significativa mayor actividad analgésica en comparación con la administración preoperatoria de la asociación dexametasona con ketorolaco.

Sin embargo, no hay diferencia significativa con relación con la tumefacción facial.

\section{CONFLICTOS DE INTERESES}

Ninguno.

\section{FUENTE DE FINANCIACIÓN}

La presente investigación no ha recibido ayudas específicas provenientes de agencias del sector público, sector comercial o entidades sin ánimo de lucro.

\section{B I B L I O G R A F Í A}

1. Prashar DV, Pahwa D, Kalia V, Jindal G, Kaur R. A comparative evaluation of the effect of diclofenac sodium with and without per-orally administered methylprednisolone on the sequelae of impacted mandibular third molar removal: A cohort randomized double-blind clinical trial. Indian J Dent. 2016;7(1):116. DOI: 10.4103/0975-962X.158187.

2. Simone JL, Jorge WA, Horliana ACRT, Canaval TG, Tortamano IP, Simone JL, et al. Comparative analysis of preemptive analgesic effect of dexamethasone and diclofenac following third molar surgery. Braz Oral Res. 2013;27(3):266-71. DOI: 10.1590/ S1806-83242013005000012.

3. Costa FWG, Esses DFS, de Barros Silva PG, Carvalho FSR, Sá CDL, Albuquerque AFM, et al. Does the Preemptive Use of Oral Nonsteroidal Anti-inflammatory Drugs Reduce Postoperative Pain in Surgical Removal of Third Molars? A Meta-analysis of Randomized Clinical Trials. Anesth Prog. 2015;62(2):57-63. DOI: 10.2344/0003-3006-62.2.57.

4. Chegini S, Dhariwal DK. Review of evidence for the use of steroids in orthognathic surgery. Br J Oral Maxillofac Surg. 2012;50(2):97-101. DOI: 10.1016/j.bjoms.2010.11.019.

5. Lima TC, Bagordakis E, Falci SGM, dos Santos CRR, Pinheiro MLP. Pre-Emptive Effect of Dexamethasone and Diclofenac Sodium Associated With Codeine on Pain, Swelling, and Tris- mus After Third Molar Surgery: A Split-Mouth, Randomized, Triple-Blind, Controlled Clinical Trial. J Oral Maxillofac Surg. 2018;76(1):60-6. DOI: 10.1016/j.joms.2017.06.012.

6. Isiordia-Espinoza M-A, Pozos-Guillen A, Martinez-Rider R, Perez-Urizar J. Comparison of the analgesic efficacy of oral ketorolac versus intramuscular tramadol after third molar surgery: A parallel, double-blind, randomized, placebo-controlled clinical trial. Med Oral Patol Oral Cir Bucal. 2016;21(5):e637-43. DOI: 10.4317/medoral.21077.

7. Fernandes IA, de Souza GM, Pinheiro MLP, Falci SGM. Intramuscular injection of dexamethasone for the control of pain, swelling, and trismus after third molar surgery: a systematic review and meta-analysis. Int J Oral Maxillofac Surg. 2019;48(5):659-68. DOI: 10.1016/j.ijom.2018.09.014.

8. Shamseer L, Hopewell S, Altman DG, Moher D, Schulz KF. Update on the endorsement of CONSORT by high impact factor journals: a survey of journal "Instructions to Authors" in 2014. Trials. 2016;17(1):301. DOI: 10.1186/s13063-016-1408-z.

9. General Assembly of the World Medical Association. World Medical Association Declaration of Helsinki: ethical principles for medical research involving human subjects. J Am Coll Dent. 2014;81(3):14-8.

10. Gbotolorun OM, Arotiba GT, Ladeinde AL. Assessment of Factors Associated With Surgical Difficulty in Impacted Mandibular Third Molar Extraction. Journal of Oral and Maxillofacial Surgery. 2007;65(10):1977-83. DOI: 10.1016/j.joms.2006.11.030.

11. Falci SGM, Lima TC, Martins CC, dos Santos CRR, Pinheiro MLP. Preemptive Effect of Dexamethasone in Third-Molar Surgery: A Meta-Analysis. Anesth Prog. Fall 2017;64(3):136-43. DOI: 10.2344/anpr-64-05-08.

12. Theken KN. Variability in analgesic response to non-steroidal anti-inflammatory drugs. Prostaglandins Other Lipid Mediat. 2018;139:63-70. DOI: 10.1016/j.prostaglandins.2018.10.005.

13. Gorecki P, Rainsford KD, Taneja P, Bulsara Y, Pearson D, Saund D, et al. Submucosal Diclofenac for Acute Postoperative Pain in Third Molar Surgery: A Randomized, Controlled Clinical Trial. J Dent Res. 2018;97(4):381-7. DOI: 10.1177/0022034517744207.

14. Grant GM, Mehlisch DR. Intranasal ketorolac for pain secondary to third molar impaction surgery: a randomized, double-blind, placebo-controlled trial. J Oral Maxillofac Surg. 2010;68(5):102531. DOI: 10.1016/j.joms.2009.10.023.

15. Ong KS, Seymour RA, Chen FG, Ho VCL. Preoperative ketorolac has a preemptive effect for postoperative third molar surgical pain. Int J Oral Maxillofac Surg. 2004;33(8):771-6. DOI: 10.1016/j. ijom.2004.01.020.

16. Aminoshariae A, Kulild JC, Donaldson M. Short-term use of nonsteroidal anti-inflammatory drugs and adverse effects: An updated systematic review. J Am Dent Assoc. 2016;147(2):98110. DOI: 10.1016/j.adaj.2015.07.020.

17. Bamgbose BO, Akinwande JA, Adeyemo WL, Ladeinde AL, Arotiba GT, Ogunlewe MO. Effects of co-administered dexamethasone and diclofenac potassium on pain, swelling and trismus following third molar surgery. Head Face Med. 2005;1(1):11. DOI: 10.1186/1746-160X-1-11.

18. Hernández-Díaz S, Rodríguez LAG. Steroids and Risk of Upper Gastrointestinal Complications. Am J Epidemiol. 2001;153(11):1089-93. DOI: 10.1093/aje/153.11.1089.

19. Buyukkurt MC, Gungormus M, Kaya O. The Effect of a Single Dose Prednisolone With and Without Diclofenac on Pain, Trismus, and Swelling After Removal of Mandibular Third Molars. J Oral Maxillofac Surg. 2006;64(12):1761-6. DOI: 10.1016/j. joms.2005.11.107.

20. Singh TK, Mathur R, Passi D, Talathi AA, Thakur SS, Singh N, et al. Comparative Evaluation of Analgesic Efficacy of Oral Ketorolac and Tramadol after Impacted Mandibular Third Molar Surgery: A Short Clinical Study with Literature Review. Annals of Medical and Health Sciences Research [Internet]. 2018 [cited 2019 Jan 12]; Available from: https://www.amhsr.org/abstract/comparative-evaluation-of-analgesic-efficacy-of-oral- 
ketorolac-and-tramadol-after-impacted-mandibular-thirdmolar-surger-4636.html

21. Mony D, Kulkarni D, Shetty L. Comparative Evaluation of Preemptive Analgesic Effect of Injected Intramuscular Diclofenac and Ketorolac after Third Molar Surgery- A Randomized Controlled Trial. J Clin Diagn Res. 2016;10(6):ZC102-6. DOI: 10.7860/JCDR/2016/17696.8045.

22. Medeiros-Albuquerque AF, Sampaio-Melo CM, Studart-Soares EC, Rodrigues-Ribeiro T, Roriz-Fonteles CS, Alves-Pereira KM, et al. Preemptive analgesia-related gene and protein expression in third molar surgeries under non steroidal anti-inflammatory drug protocols: A PROSPERO-registered systematic review of clinical studies. Med Oral Patol Oral Cir Bucal. 2018;23(6):e723-e732. DOI: 10.4317/medoral.22576.

23. Khan AA, Iadarola M, Yang HT, Dionne RA. Expression of COX1 and COX-2 in a Clinical Model of Acute Inflammation. J Pain. 2007;8:349-54. DOI: 10.1016/j.jpain.2006.10.004.

24. Lee YS, Kim H, Wu TX, Wang XM, Dionne RA. Genetically mediated interindividual variation in analgesic responses to cyclooxygenase inhibitory drugs. Clin Pharmacol Ther. 2006;79:407-18. DOI: 10.1016/j.clpt.2006.01.013.

25. Schünemann HJ, Best D, Vist G, Oxman AD. Letters, numbers, symbols and words: how to communicate grades of evidence and recommendations. CMAJ. 2003;169:677-80. 